\title{
Exponential and polynomial decay for a laminated beam with Fourier's type heat conduction
}

\author{
Wenjun Liu and Weifan Zhao \\ College of Mathematics and Statistics, Nanjing University of Information Science and Technology, \\ Nanjing 210044, China. E-mail: wjliu@nuist.edu.cn.
}

In this paper, we study the well-posedness and the asymptotic behavior of a onedimensional laminated beam system, where the heat conduction is given by Fourier's law effective in the rotation angle displacements. We show that the system is wellposed by using the Hille-Yosida theorem and prove that the system is exponentially stable if and only if the wave speeds are equal. Furthermore, we show that the system is polynomially stable provided that the wave speeds are not equal.

Keywords: laminated beam, Fourier's law, exponential stability, lack of exponential stability, polynomial stability.

\section{Introduction}

With the increasing demand of advanced performance, the vibration suppression of the laminated beams has been one of the main research topics in smart materials and structures. These composite laminates usually have superior structural properties such as adaptability. The design of their piezoelectric materials can be used as both actuators and sensors [1]. Hansen and Spies in [2] derived the mathematical model for two-layered beams with structural damping due to the interfacial slip, the system is given by the following equations:

$$
\begin{cases}\rho \varphi_{t t}+G\left(\psi-\varphi_{x}\right)_{x}=0, & (x, t) \in(0,1) \times(0,+\infty), \\ I_{\rho}(3 w-\psi)_{t t}-G\left(\psi-\varphi_{x}\right)-D(3 w-\psi)_{x x}=0, & (x, t) \in(0,1) \times(0,+\infty), \\ 3 I_{\rho} w_{t t}+3 G\left(\psi-\varphi_{x}\right)+4 \gamma w+4 \beta w_{t}-3 D w_{x x}=0, & (x, t) \in(0,1) \times(0,+\infty),\end{cases}
$$

where $\rho, G, I_{\rho}, D, \gamma, \beta$ are positive constant coefficients, $\rho$ is the density of the beams, $G$ is the shear stiffness, $I_{\rho}$ is the mass moment of inertia, $D$ is the flexural rigidity, $\gamma$ is the adhesive stiffness of the beams, and $\beta$ is the adhesive damping parameter. The function $\varphi$ denotes the transverse displacement of the beam which departs from its equilibrium position, $\psi$ represents the rotation angle, $w$ is proportional to the amount of slip along the interface at time $t$ and longitudinal spatial variable $x, 3 w-\psi$ denotes the effective rotation angle, $(1.1)_{3}$ describes the dynamics of the slip.

In recent years, an increasing interest has been developed to determine the asymptotic behavior of the solution of several laminated beam problems. For example, Wang et al. [1] considered system (1.1) with the cantilever boundary conditions and two different wave speeds $(\sqrt{G / \rho}$ and $\left.\sqrt{D / I_{\rho}}\right)$. The authors proved the well-posedness and pointed out that system (1.1) can obtain the asymptotic stability but it does not reach the exponential stability due to the action of the slip $w$. Furthermore, to achieve the exponential decay result, the authors added an additional boundary control such that the boundary conditions become

$$
\begin{gathered}
\varphi(0, t)=\xi(0, t)=w(0, t)=0, w_{x}(1, t)=0, \\
3 w(1, t)-\xi(1, t)-\varphi_{x}(1, t)=u_{1}(t):=k_{1} \varphi_{t}(1, t),
\end{gathered}
$$




$$
\xi_{x}(1, t)=u_{2}(t):=-k_{2} \xi_{t}(1, t),
$$

where $\xi=3 w-\psi$. Cao et al. [3] considered the system (1.1) with following boundary conditions

$$
\begin{gathered}
\psi(0, t)-\varphi_{x}(0, t)=u_{1}(t):=-k_{1} \varphi_{t}(0, t)-\varphi(0, t), \\
3 w_{x}(1, t)-\psi_{x}(1, t)=u_{2}(t):=-k_{2} \xi_{t}(1, t)-\xi(1, t),
\end{gathered}
$$

where $\xi=3 w-\psi$. The authors obtained an exponential stability result provided $k_{1} \neq \sqrt{\rho / G}$ and $k_{2} \neq \sqrt{I_{\rho} / D}$. More importantly, the authors proved that the dominant part of the system is itself exponentially stable. Raposo [4] considered system (1.1) with two frictional dampings of the form

$$
\left\{\begin{array}{lr}
\rho \varphi_{t t}+G\left(\psi-\varphi_{x}\right)_{x}+k_{1} \varphi_{t}=0, & (x, t) \in(0,1) \times(0,+\infty), \\
I_{\rho}(3 w-\psi)_{t t}-G\left(\psi-\varphi_{x}\right)-D(3 w-\psi)_{x x}+k_{2}(3 w-\psi)_{t}=0, & (x, t) \in(0,1) \times(0,+\infty), \\
3 I_{\rho} w_{t t}+3 G\left(\psi-\varphi_{x}\right)+4 \gamma w+4 \beta w_{t}-3 D w_{x x}=0, & (x, t) \in(0,1) \times(0,+\infty)
\end{array}\right.
$$

and obtained the exponential decay result under appropriate initial and boundary conditions.

It is easy to find that if the slip $w$ is assumed to be identically zero, then the first two equations of system (1.1) can be reduced exactly to the Timoshenko beam system. For the case of the Timoshenko beam with Fourier's law, many authors have shown various decay estimates depending on the wave speeds. Rivera and Racke [5] studied the Timoshenko system with thermoelastic dissipation, i.e.,

$$
\left\{\begin{array}{lr}
\rho_{1} \varphi_{t t}-k\left(\varphi_{x}+\psi\right)_{x}=0, & (x, t) \in(0, L) \times(0,+\infty), \\
\rho_{2} \psi_{t t}-b \psi_{x x}+k\left(\varphi_{x}+\psi\right)+\gamma \theta_{x}=0, & (x, t) \in(0, L) \times(0,+\infty), \\
\rho_{3} \theta_{t}-\kappa \theta_{x x}+\gamma \psi_{t x}=0, & (x, t) \in(0, L) \times(0,+\infty),
\end{array}\right.
$$

with positive constants $\rho_{1}, \rho_{2}, \rho_{3}, k, b, \gamma, \kappa$. The authors showed that the exponential stability holds if and only if the wave speeds are equal $\left(\frac{k}{\rho_{1}}=\frac{b}{\rho_{2}}\right)$. Júnior and Rivera [6] considered a new coupling to the thermoelastic Timoshenko beam of the form

$$
\begin{cases}\rho_{1} \varphi_{t t}-k\left(\varphi_{x}+\psi\right)_{x}+\sigma \theta_{x}=0, & (x, t) \in(0, L) \times(0,+\infty), \\ \rho_{2} \psi_{t t}-b \psi_{x x}+k\left(\varphi_{x}+\psi\right)-\sigma \theta=0, & (x, t) \in(0, L) \times(0,+\infty), \\ \rho_{3} \theta_{t}-\gamma \theta_{x x}+\sigma\left(\varphi_{x}+\psi\right)_{t}=0, & (x, t) \in(0, L) \times(0,+\infty) .\end{cases}
$$

The authors showed this system is exponentially stable if and only if the wave speeds are equal $\left(\frac{k}{\rho_{1}}=\frac{b}{\rho_{2}}\right)$. On the contrary, the authors obtained the polynomially stable depending on the different boundary conditions. For system (1.4) with Dirichlet boundary conditions

$$
\varphi(t, 0)=\varphi(t, L)=\psi(t, 0)=\psi(t, L)=\theta(t, 0)=\theta(t, L)=0,
$$

the authors obtained that the semigroup decay as $\frac{1}{\sqrt[4]{t}}$. For system (1.4) with Dirichlet-Neumann boundary conditions

$$
\varphi(t, 0)=\varphi(t, L)=\psi_{x}(t, 0)=\psi_{x}(t, L)=\theta_{x}(t, 0)=\theta_{x}(t, L)=0,
$$

the authors obtained that the semigroup decay as $\frac{1}{\sqrt{t}}$. We refer the reader to $[7,8,9,10,11,12$, $13,14,15,16,17]$, for some other related results. 
Motivated by the above results, we intend to study the well-posedness and the asymptotic stability of the laminated beam system where the heat flux is given by Fourier's law. The system is written as

$$
\left\{\begin{array}{lr}
\rho \varphi_{t t}+G\left(\psi-\varphi_{x}\right)_{x}=0, & (x, t) \in(0,1) \times(0,+\infty), \\
I_{\rho}(3 w-\psi)_{t t}-G\left(\psi-\varphi_{x}\right)-D(3 w-\psi)_{x x}+\sigma \theta_{x}=0, & (x, t) \in(0,1) \times(0,+\infty), \\
I_{\rho} w_{t t}+G\left(\psi-\varphi_{x}\right)+\frac{4}{3} \gamma w+\frac{4}{3} \beta w_{t}-D w_{x x}=0, & (x, t) \in(0,1) \times(0,+\infty), \\
k \theta_{t}-\tau \theta_{x x}+\sigma(3 w-\psi)_{t x}=0, & (x, t) \in(0,1) \times(0,+\infty),
\end{array}\right.
$$

where $\rho, G, I_{\rho}, D, \sigma, \gamma, \beta, k, \tau$ are positive constant coefficients. We consider following initial and boundary conditions

$$
\begin{cases}\varphi(x, 0)=\varphi_{0}(x), \varphi_{t}(x, 0)=\varphi_{1}(x), & x \in[0,1], \\ \psi(x, 0)=\psi_{0}(x), \psi_{t}(x, 0)=\psi_{1}(x), & x \in[0,1], \\ w(x, 0)=w_{0}(x), w_{t}(x, 0)=w_{1}(x), & x \in[0,1], \\ \theta(x, 0)=\theta_{0}(x), & x \in[0,1], \\ \varphi(0, t)=\psi_{x}(0, t)=w_{x}(0, t)=\theta(0, t)=0, & t \in[0,+\infty), \\ \varphi_{x}(1, t)=\psi(1, t)=w(1, t)=\theta_{x}(1, t)=0, & t \in[0,+\infty) .\end{cases}
$$

By using Hille-Yosida theorem, we first prove the well-posedness result. By using the perturbed energy method, we then establish the exponential result if and only if $\frac{\rho}{G}=\frac{I_{\rho}}{D}$ and the polynomial stability if $\frac{\rho}{G} \neq \frac{I_{\rho}}{D}$. Furthermore, by using Gearhart-Herbst-Prüss-Huang theorem, we obtain the lack of exponential stability. The main difficulty in carry out this paper is the appearance for the Fourier's law of heat conduction. For this purpose, we use the appropriated multiplies and energy method to build an equivalent Lyapunov functional.

We now briefly sketch the outline of the paper. In Section 2, we state and prove the wellposedness of problem (1.5)-(1.6). In Section 3, we establish an exponential stability result of the energy. In Section 4, the lack of exponential stability has been studied. Finally, Section 5 is devoted to the statement and proof of the polynomial stability.

\section{The well-posedness}

In this Section, we prove the well-posedness of problem (1.5)-(1.6) by using Hille-Yosida theorem. Firstly, we introduce the vector function

$$
U=\left(\varphi, \varphi_{t}, 3 w-\psi,(3 w-\psi)_{t}, w, w_{t}, \theta\right)^{T} .
$$

Then system (1.5)-(1.6) can be written as

$$
\left\{\begin{array}{l}
\partial_{t} U=\mathscr{A} U \\
U(x, 0)=U^{0}(x)=\left(\varphi_{0}, \varphi_{1}, 3 w_{0}-\psi_{0}, 3 w_{1}-\psi_{1}, w_{0}, w_{1}, \theta_{0}\right)^{T},
\end{array}\right.
$$


where $\mathscr{A}$ is a linear operator defined by

$$
\mathscr{A} U=\left(\begin{array}{c}
\varphi_{t} \\
-\frac{G}{\rho}\left(\psi-\varphi_{x}\right)_{x} \\
(3 w-\psi)_{t} \\
\frac{G}{I_{\rho}}\left(\psi-\varphi_{x}\right)+\frac{D}{I_{\rho}}(3 w-\psi)_{x x}-\frac{\sigma}{I_{\rho}} \theta_{x} \\
w_{t} \\
-\frac{G}{I_{\rho}}\left(\psi_{-} \varphi_{x}\right)-\frac{4 \gamma}{3 I_{\rho}} w-\frac{4 \beta}{3 I_{\rho}} w_{t}+\frac{D}{I_{\rho}} w_{x x} \\
\frac{\tau}{k} \theta_{x x}-\frac{\sigma}{k}(3 w-\psi)_{t x}
\end{array}\right) .
$$

We consider the following spaces:

$$
\begin{aligned}
H_{*}^{1}(0,1)=\left\{\eta \mid \eta \in H^{1}(0,1): \eta(0)=0\right\}, & \tilde{H}_{*}^{1}(0,1)=\left\{\eta \mid \eta \in H^{1}(0,1): \eta(1)=0\right\}, \\
& H_{*}^{2}(0,1)=H^{2}(0,1) \cap H_{*}^{1}(0,1), \quad \tilde{H}_{*}^{2}(0,1)=H^{2}(0,1) \cap \tilde{H}_{*}^{1}(0,1),
\end{aligned}
$$

and

$$
\mathscr{H}=H_{*}^{1}(0,1) \times L^{2}(0,1) \times \tilde{H}_{*}^{1}(0,1) \times L^{2}(0,1) \times \tilde{H}_{*}^{1}(0,1) \times L^{2}(0,1) \times L^{2}(0,1),
$$

equipped with the inner product

$$
\begin{aligned}
(U, \tilde{U})_{\mathscr{H}}= & \rho \int_{0}^{1} \varphi_{t} \tilde{\varphi}_{t} \mathrm{~d} x+I_{\rho} \int_{0}^{1}(3 w-\psi)_{t}(3 \tilde{w}-\tilde{\psi})_{t} \mathrm{~d} x+3 I_{\rho} \int_{0}^{1} w_{t} \tilde{w}_{t} \mathrm{~d} x+k \int_{0}^{1} \theta \tilde{\theta} \mathrm{d} x \\
& +G \int_{0}^{1}\left(\psi-\varphi_{x}\right)\left(\tilde{\psi}-\tilde{\varphi}_{x}\right) \mathrm{d} x+D \int_{0}^{1}(3 w-\psi)_{x}(3 \tilde{w}-\tilde{\psi})_{x} \mathrm{~d} x+4 \gamma \int_{0}^{1} w \tilde{w} \mathrm{~d} x \\
& +3 D \int_{0}^{1} w_{x} \tilde{w}_{x} \mathrm{~d} x
\end{aligned}
$$

Then, the domain of $\mathscr{A}$ is given by

$$
\begin{aligned}
& D(\mathscr{A})=\left\{U \in \mathscr{H} \mid \varphi \in H_{*}^{2}(0,1), 3 w-\psi, w \in \tilde{H}_{*}^{2}(0,1), \theta \in H_{*}^{1}(0,1), \varphi_{t} \in H_{*}^{1}(0,1),\right. \\
&\left.3 w_{t}-\psi_{t}, w_{t} \in \tilde{H}_{*}^{1}(0,1), \varphi_{x}(1, t)=0, \psi_{x}(0, t)=w_{x}(0, t)=0\right\} .
\end{aligned}
$$

The well-posedness of problem (2.1) is ensured by

Theorem 2.1 Let $U^{0} \in \mathscr{H}$, then problem (2.1) exists a unique weak solution $U \in C\left(\mathbb{R}^{+} ; \mathscr{H}\right)$. Moreover, if $U^{0} \in D(\mathscr{A})$, then

$$
U \in C\left(\mathbb{R}^{+} ; D(\mathscr{A})\right) \cap C^{1}\left(\mathbb{R}^{+} ; \mathscr{H}\right) .
$$


Proof. To obtain the above result, we need to prove that $\mathscr{A}: D(\mathscr{A}) \rightarrow \mathscr{H}$ is a maximal monotone operator. For this purpose, we need the following two steps: $\mathscr{A}$ is dissipative and $I d-\mathscr{A}$ is surjective.

Step 1. $\mathscr{A}$ is dissipative.

For any $U \in D(\mathscr{A})$, by using the inner product and integration by parts, we can imply that

$$
\langle\mathscr{A} U, U\rangle_{\mathscr{H}}=-\tau \int_{0}^{1} \theta_{x}^{2} \mathrm{~d} x-4 \beta \int_{0}^{1} w_{t}^{2} \mathrm{~d} x \leq 0 .
$$

Hence, $\mathscr{A}$ is a dissipative operator.

Step 2. $I d-\mathscr{A}$ is surjective.

To prove that the operator $I d-\mathscr{A}$ is surjective, that is, for any $F=\left(f_{1}, \cdots, f_{7}\right) \in \mathscr{H}$, there exists $V=\left(v_{1}, \cdots, v_{7}\right) \in D(\mathscr{A})$ satisfying

$$
(I d-\mathscr{A}) V=F
$$

which is equivalent to

$$
\left\{\begin{array}{l}
v_{1}-v_{2}=f_{1} \\
\rho v_{2}-G \partial_{x x} v_{1}-G \partial_{x} v_{3}+3 G \partial_{x} v_{5}=\rho f_{2} \\
v_{3}-v_{4}=f_{3} \\
I_{\rho} v_{4}+G \partial_{x} v_{1}+G v_{3}-D \partial_{x x} v_{3}-3 G v_{5}+\sigma \partial_{x} v_{7}=I_{\rho} f_{4} \\
v_{5}-v_{6}=f_{5} \\
\left(I_{\rho}+\frac{4 \beta}{3}\right) v_{6}-G \partial_{x} v_{1}-G v_{3}+\left(3 G+\frac{4 \gamma}{3}\right) v_{5}-D \partial_{x x} v_{5}=I_{\rho} f_{6} \\
k v_{7}-\tau \partial_{x x} v_{7}+\sigma \partial_{x} v_{4}=k f_{7}
\end{array}\right.
$$

$(2.5)_{1},(2.5)_{3}$ and $(2.5)_{5}$ give

$$
\left\{\begin{array}{l}
v_{2}=v_{1}-f_{1}, \\
v_{4}=v_{3}-f_{3}, \\
v_{6}=v_{5}-f_{5} .
\end{array}\right.
$$

Inserting (2.6) into $(2.5)_{2},(2.5)_{4},(2.5)_{6}$ and $(2.5)_{7}$, we get

$$
\left\{\begin{array}{l}
\rho v_{1}-G \partial_{x x} v_{1}-G \partial_{x} v_{3}+3 G \partial_{x} v_{5}=\rho\left(f_{1}+f_{2}\right) \\
\left(I_{\rho}+G\right) v_{3}+G \partial_{x} v_{1}-D \partial_{x x} v_{3}-3 G v_{5}+\sigma \partial_{x} v_{7}=I_{\rho}\left(f_{3}+f_{4}\right) \\
\left(I_{\rho}+3 G+\frac{4 \beta}{3}+\frac{4 \gamma}{3}\right) v_{5}-G \partial_{x} v_{1}-G v_{3}-D \partial_{x x} v_{5}=I_{\rho}\left(f_{5}+f_{6}\right)+\frac{4 \beta}{3} f_{5} \\
k v_{7}+\sigma \partial_{x} v_{3}-\tau \partial_{x x} v_{7}=\sigma \partial_{x} f_{3}+k f_{7}
\end{array}\right.
$$

Multiplying $(2.7)_{1}-(2.7)_{4}$ by $\tilde{v}_{1}, \tilde{v}_{3}, 3 \tilde{v}_{5}$ and $\tilde{v}_{7}$ respectively, and integrating over $(0,1)$, we arrive 
at

$$
\left\{\begin{array}{l}
\int_{0}^{1} \rho v_{1} \tilde{v}_{1} \mathrm{~d} x-\int_{0}^{1} G \partial_{x x} v_{1} \tilde{v}_{1} \mathrm{~d} x-\int_{0}^{1} G \partial_{x} v_{3} \tilde{v}_{1} \mathrm{~d} x+\int_{0}^{1} 3 G \partial_{x} v_{5} \tilde{v}_{1} \mathrm{~d} x=\int_{0}^{1} \rho\left(f_{1}+f_{2}\right) \tilde{v}_{1} \mathrm{~d} x \\
\int_{0}^{1}\left(I_{\rho}+G\right) v_{3} \tilde{v}_{3} \mathrm{~d} x+\int_{0}^{1} G \partial_{x} v_{1} \tilde{v}_{3} \mathrm{~d} x-\int_{0}^{1} D \partial_{x x} v_{3} \tilde{v}_{3} \mathrm{~d} x-\int_{0}^{1} 3 G v_{5} \tilde{v}_{3} \mathrm{~d} x+\int_{0}^{1} \sigma \partial_{x} v_{7} \tilde{v}_{3} \mathrm{~d} x \\
=\int_{0}^{1} I_{\rho}\left(f_{3}+f_{4}\right) \tilde{v}_{3} \mathrm{~d} x \\
\int_{0}^{1}\left(3 I_{\rho}+9 G+4 \beta+4 \gamma\right) v_{5} \tilde{v}_{5} \mathrm{~d} x-\int_{0}^{1} 3 G \partial_{x} v_{1} \tilde{v}_{5} \mathrm{~d} x-\int_{0}^{1} 3 G v_{3} \tilde{v}_{5} \mathrm{~d} x-\int_{0}^{1} 3 D \partial_{x x} v_{5} \tilde{v}_{5} \mathrm{~d} x \\
=\int_{0}^{1} 3 I_{\rho}\left(f_{5}+f_{6}\right) \tilde{v}_{5} \mathrm{~d} x+\int_{0}^{1} 4 \beta f_{5} \tilde{v}_{5} \mathrm{~d} x \\
\int_{0}^{1} k v_{7} \tilde{v}_{7} \mathrm{~d} x+\int_{0}^{1} \sigma \partial_{x} v_{3} \tilde{v}_{7} \mathrm{~d} x-\int_{0}^{1} \tau \partial_{x x} v_{7} \tilde{v}_{7} \mathrm{~d} x=\int_{0}^{1} \sigma \partial_{x} f_{3} \tilde{v}_{7} \mathrm{~d} x+\int_{0}^{1} k f_{7} \tilde{v}_{7} \mathrm{~d} x
\end{array}\right.
$$

The sum of the equations in (2.8) gives the following variational formulation:

$$
\begin{gathered}
a\left(\left(v_{1}, v_{3}, v_{5}, v_{7}\right)^{T},\left(\tilde{v}_{1}, \tilde{v}_{3}, \tilde{v}_{5}, \tilde{v}_{7}\right)^{T}\right)=\tilde{a}\left(\left(\tilde{v}_{1}, \tilde{v}_{3}, \tilde{v}_{5}, \tilde{v}_{7}\right)^{T}\right), \\
\forall\left(\tilde{v}_{1}, \tilde{v}_{3}, \tilde{v}_{5}, \tilde{v}_{7}\right)^{T} \in H_{*}^{1}(0,1) \times \tilde{H}_{*}^{1}(0,1) \times \tilde{H}_{*}^{1}(0,1) \times L^{2}(0,1),
\end{gathered}
$$

where

$$
\begin{aligned}
& a\left(\left(v_{1}, v_{3}, v_{5}, v_{7}\right)^{T},\left(\tilde{v}_{1}, \tilde{v}_{3}, \tilde{v}_{5}, \tilde{v}_{7}\right)^{T}\right) \\
= & \int_{0}^{1} G\left(-\partial_{x} v_{1}-v_{3}+3 v_{5}\right)\left(-\partial_{x} \tilde{v}_{1}-\tilde{v}_{3}+3 \tilde{v}_{5}\right) \mathrm{d} x+\int_{0}^{1} \rho v_{1} \tilde{v}_{1} \mathrm{~d} x+\int_{0}^{1} I_{\rho} v_{3} \tilde{v}_{3} \mathrm{~d} x \\
& +\int_{0}^{1}\left(3 I_{\rho}+4 \gamma+4 \beta\right) v_{5} \tilde{v}_{5} \mathrm{~d} x+\int_{0}^{1} k v_{7} \tilde{v}_{7} \mathrm{~d} x+\int_{0}^{1} D \partial_{x} v_{3} \partial_{x} \tilde{v}_{3} \mathrm{~d} x+\int_{0}^{1} 3 D \partial_{x} v_{5} \partial_{x} \tilde{v}_{5} \mathrm{~d} x \\
& +\tau \int_{0}^{1} \partial_{x} v_{7} \partial_{x} \tilde{v}_{7} \mathrm{~d} x+\sigma \int_{0}^{1}\left(\partial_{x} v_{7}\right) \tilde{v}_{3} \mathrm{~d} x+\sigma \int_{0}^{1}\left(\partial_{x} v_{3}\right) \tilde{v}_{7} \mathrm{~d} x
\end{aligned}
$$

and

$$
\begin{aligned}
& \tilde{a}\left(\left(\tilde{v}_{1}, \tilde{v}_{3}, \tilde{v}_{5}, \tilde{v}_{7}\right)^{T}\right) \\
= & \int_{0}^{1}\left(\rho\left(f_{1}+f_{2}\right) \tilde{v}_{1}+I_{\rho}\left(f_{3}+f_{4}\right) \tilde{v}_{3}+3 I_{\rho}\left(f_{5}+f_{6}\right) \tilde{v}_{5}+4 \beta f_{5} \tilde{v}_{5}+\sigma \partial_{x} f_{3} \tilde{v}_{7}+k f_{7} \tilde{v}_{7}\right) \mathrm{d} x .
\end{aligned}
$$

Now, we introduce the Hilbert space $V=H_{*}^{1}(0,1) \times \tilde{H}_{*}^{1}(0,1) \times \tilde{H}_{*}^{1}(0,1) \times L^{2}(0,1)$ equipped with the norm

$$
\left\|\left(v_{1}, v_{3}, v_{5}, v_{7}\right)\right\|_{V}^{2}=\left\|-\partial_{x} v_{1}-v_{3}+3 v_{5}\right\|_{2}^{2}+\left\|v_{1}\right\|_{2}^{2}+\left\|\partial_{x} v_{3}\right\|_{2}^{2}+\left\|\partial_{x} v_{5}\right\|_{2}^{2}+\left\|\partial_{x} v_{7}\right\|_{2}^{2}
$$

It is clear that $a(\cdot, \cdot)$ and $\tilde{a}(\cdot)$ are bounded. Furthermore, we can obtain that there exists a positive constant $m$ such that

$$
\begin{aligned}
& a\left(\left(v_{1}, v_{3}, v_{5}, v_{7}\right)^{T},\left(v_{1}, v_{3}, v_{5}, v_{7}\right)^{T}\right) \\
= & \int_{0}^{1} G\left(-\partial_{x} v_{1}-v_{3}+3 v_{5}\right)^{2} \mathrm{~d} x+\int_{0}^{1} \rho v_{1}^{2} \mathrm{~d} x+\int_{0}^{1} I_{\rho} v_{3}^{2} \mathrm{~d} x+\int_{0}^{1}\left(3 I_{\rho}+4 \gamma+4 \beta\right) v_{5}^{2} \mathrm{~d} x
\end{aligned}
$$




$$
\begin{aligned}
& \quad+\int_{0}^{1} k v_{7}^{2} \mathrm{~d} x+\int_{0}^{1} D\left(\partial_{x} v_{3}\right)^{2} \mathrm{~d} x+\int_{0}^{1} 3 D\left(\partial_{x} v_{5}\right)^{2} \mathrm{~d} x+\tau \int_{0}^{1}\left(\partial_{x} v_{7}\right)^{2} \mathrm{~d} x \\
& \geq m\left\|\left(v_{1}, v_{3}, v_{5}, v_{7}\right)\right\|_{V}^{2},
\end{aligned}
$$

which implies that $a(\cdot, \cdot)$ is coercive.

Hence, we assert that $a(\cdot, \cdot)$ is a bilinear continuous coercive form on $V \times V$, and $\tilde{a}(\cdot)$ is a linear continuous form on $V$. Applying the Lax-Milgram theorem [18], we obtain that (2.8) has a unique solution $\left(v_{1}, v_{3}, v_{5}, v_{7}\right)^{T} \in V$. Then, by substituting $v_{1}, v_{3}, v_{5}$ into (2.6), we obtain

$$
v_{2} \in H_{*}^{1}(0,1), v_{4} \in \tilde{H}_{*}^{1}(0,1), v_{6} \in \tilde{H}_{*}^{1}(0,1) .
$$

Next, it remains to show that

$$
v_{1} \in H_{*}^{2}(0,1), v_{3} \in \tilde{H}_{*}^{2}(0,1), v_{5} \in \tilde{H}_{*}^{2}(0,1), v_{7} \in H_{*}^{1}(0,1), \partial_{x} v_{1}(1)=\partial_{x} v_{3}(0)=\partial_{x} v_{5}(0) .
$$

Furthermore, if $\left(\tilde{v}_{3}, \tilde{v}_{5}, \tilde{v}_{7}\right) \equiv(0,0,0) \in \tilde{H}_{*}^{1}(0,1) \times \tilde{H}_{*}^{1}(0,1) \times L^{2}(0,1)$, then $(2.9)$ reduces to

$$
\int_{0}^{1} G \partial_{x x} v_{1} \tilde{v}_{1} \mathrm{~d} x=\int_{0}^{1} \rho v_{1} \tilde{v}_{1} \mathrm{~d} x-\int_{0}^{1} G \partial_{x} v_{3} \tilde{v}_{1} \mathrm{~d} x+\int_{0}^{1} 3 G \partial_{x} v_{5} \tilde{v}_{1} \mathrm{~d} x-\int_{0}^{1} \rho\left(f_{1}+f_{2}\right) \tilde{v}_{1} \mathrm{~d} x
$$

for all $\tilde{v}_{1} \in H_{*}^{1}(0,1)$, which implies

$$
G \partial_{x x} v_{1}=\rho v_{1}-G \partial_{x} v_{3}+3 G \partial_{x} v_{5}-\rho\left(f_{1}+f_{2}\right) \in L^{2}(0,1) .
$$

Thus, by the $L^{2}$ theory for the linear elliptic equations, we obtain that

$$
v_{1} \in H_{*}^{2}(0,1) .
$$

Moreover, (2.10) is also true for any $\phi \in C^{1}([0,1]) \subset H_{*}^{1}(0,1)(\phi(0)=0)$. Hence, we get

$$
\int_{0}^{1} G \partial_{x} v_{1} \partial_{x} \phi \mathrm{d} x+\int_{0}^{1} \rho v_{1} \phi \mathrm{d} x-\int_{0}^{1} G\left(\partial_{x} v_{3}\right) \phi \mathrm{d} x+\int_{0}^{1} 3 G\left(\partial_{x} v_{5}\right) \phi \mathrm{d} x=\int_{0}^{1} \rho\left(f_{1}+f_{2}\right) \phi \mathrm{d} x .
$$

By using the integration by parts, we have

$$
\partial_{x} v_{1}(1) \phi(1)=0, \forall \phi \in C^{1}([0,1]), \phi(0)=0 .
$$

Therefore,

$$
\partial_{x} v_{1}(1)=0
$$

In the same way, we get

$$
v_{3} \in \tilde{H}_{*}^{2}(0,1), v_{5} \in \tilde{H}_{*}^{2}(0,1), v_{7} \in H_{*}^{1}(0,1), \partial_{x} v_{3}(0)=\partial_{x} v_{5}(0)=0 .
$$

Finally, the application of the classical regularity theory for linear elliptic equations guarantees the existence of unique solution $V \in D(\mathscr{A})$ which satisfies (2.4). Hence, the operator $I d-\mathscr{A}$ is surjective. Moreover, it is easy to see that $D(\mathscr{A})$ is dense in $\mathscr{H}$.

At last, by Hille-Yosida theorem (see $[19,20]$ ) we have the well-posedness result stated in Theorem 2.1. 


\section{Exponential stability}

In this Section, we prove the exponential decay for problem (1.5)-(1.6). It will be achieved by using the perturbed energy method. We define the energy functional $E(t)$ as

$$
\begin{aligned}
E(t):=E(u(t))= & \frac{1}{2}\left(\rho \int_{0}^{1} \varphi_{t}^{2} \mathrm{~d} x+I_{\rho} \int_{0}^{1}\left(3 w_{t}-\psi_{t}\right)^{2} \mathrm{~d} x+3 I_{\rho} \int_{0}^{1} w_{t}^{2} \mathrm{~d} x+G \int_{0}^{1}\left(\psi-\varphi_{x}\right)^{2} \mathrm{~d} x\right. \\
& \left.+D \int_{0}^{1}\left(3 w_{x}-\psi_{x}\right)^{2} \mathrm{~d} x+3 D \int_{0}^{1} w_{x}^{2} \mathrm{~d} x+4 \gamma \int_{0}^{1} w^{2} \mathrm{~d} x+k \int_{0}^{1} \theta^{2} \mathrm{~d} x\right)
\end{aligned}
$$

If the wave speeds are equal, we have the following exponentially stable result.

Theorem 3.1 Assume that $\frac{\rho}{G}=\frac{I_{\rho}}{D}$ hold. Let $U^{0} \in \mathscr{H}$, then there exists positive constants $c_{0}, c_{1}$ such that the energy $E(t)$ associated with problem (1.5)-(1.6) satisfies

$$
E(t) \leq c_{0} e^{-c_{1} t}, t \geq 0 .
$$

To prove our this result, we will state and prove some useful lemmas in advance.

Lemma 3.2 Let $(\varphi, \psi, w, \theta)$ be the solution of (1.5)-(1.6). Then the energy functional satisfies

$$
\frac{d}{d t} E(t)=-4 \beta \int_{0}^{1} w_{t}^{2} \mathrm{~d} x-\tau \int_{0}^{1} \theta_{x}^{2} \mathrm{~d} x \leq 0, \forall t \geq 0 .
$$

Proof. First, multiplying $(1.5)_{1}$ by $\varphi_{t}$, integrating over $(0,1)$, using integration by parts and the boundary conditions in (1.6), we have

$$
\frac{d}{d t}\left\{\frac{1}{2} \rho \int_{0}^{1} \varphi_{t}^{2} \mathrm{~d} x\right\}-G \int_{0}^{1}\left(\psi-\varphi_{x}\right) \varphi_{x t} \mathrm{~d} x=0
$$

Note that

$$
\begin{aligned}
G \int_{0}^{1}\left(\psi-\varphi_{x}\right) \varphi_{x t} \mathrm{~d} x & =-G \int_{0}^{1}\left(\psi-\varphi_{x}\right)\left(\psi-\varphi_{x}-\psi\right)_{t} \mathrm{~d} x \\
& =\frac{d}{d t}\left\{-\frac{1}{2} G \int_{0}^{1}\left(\psi-\varphi_{x}\right)^{2} \mathrm{~d} x\right\}+G \int_{0}^{1}\left(\psi-\varphi_{x}\right) \psi_{t} \mathrm{~d} x
\end{aligned}
$$

Hence, equation (3.4) becomes

$$
\frac{d}{d t}\left\{\frac{1}{2}\left(\rho \int_{0}^{1} \varphi_{t}^{2} \mathrm{~d} x+G \int_{0}^{1}\left(\psi-\varphi_{x}\right)^{2} \mathrm{~d} x\right)\right\}=G \int_{0}^{1}\left(\psi-\varphi_{x}\right) \psi_{t} \mathrm{~d} x .
$$

Similarly, multiplying $(1.5)_{2},(1.5)_{3},(1.5)_{4}$ by $3(w-\psi)_{t}, 3 w_{t}, \theta$ and integrating over $(0,1)$, using integration by parts the boundary conditions in (1.6), we can get

$$
\begin{aligned}
& \frac{d}{d t}\left\{\frac{1}{2}\left(I_{\rho} \int_{0}^{1}\left(3 w_{t}-\psi_{t}\right)^{2} \mathrm{~d} x+D \int_{0}^{1}\left(3 w_{x}-\psi_{x}\right)^{2} \mathrm{~d} x\right)\right\} \\
= & G \int_{0}^{1}\left(\psi-\varphi_{x}\right)(3 w-\psi)_{t} \mathrm{~d} x-\sigma \int_{0}^{1} \theta_{x}(3 w-\psi)_{t} \mathrm{~d} x, \\
& \frac{d}{d t}\left\{\frac{1}{2}\left(3 I_{\rho} \int_{0}^{1} w_{t}^{2} \mathrm{~d} x+4 \gamma \int_{0}^{1} w^{2} \mathrm{~d} x+3 D \int_{0}^{1} w_{x}^{2} \mathrm{~d} x\right)\right\}
\end{aligned}
$$




$$
\begin{aligned}
= & -3 G \int_{0}^{1}\left(\psi-\varphi_{x}\right) w_{t} \mathrm{~d} x-4 \beta \int_{0}^{1} w_{t}^{2} \mathrm{~d} x, \\
& \frac{d}{d t}\left\{\frac{1}{2} k \int_{0}^{1} \theta^{2} \mathrm{~d} x\right\}=\sigma \int_{0}^{1}(3 w-\psi)_{t} \theta_{x} \mathrm{~d} x-\tau \int_{0}^{1} \theta_{x}^{2} \mathrm{~d} x .
\end{aligned}
$$

Finally, adding (3.5)-(3.8), we obtain (3.3), which completes the proof.

Next, in order to construct a Lyapunov functional equivalent to the energy, we will prove several lemmas with the purpose of creating negative counterparts of the terms that appear in the energy.

Lemma 3.3 Let $(\varphi, \psi, w, \theta)$ be the solution of (1.5)-(1.6). Then the functional

$$
I_{1}(t)=-\rho \int_{0}^{1} \varphi \varphi_{t} \mathrm{~d} x
$$

satisfies the estimate

$$
I_{1}^{\prime}(t) \leq-\rho \int_{0}^{1} \varphi_{t}^{2} \mathrm{~d} x+\left(G+\varepsilon_{1}\right) \int_{0}^{1}\left(\psi-\varphi_{x}\right)^{2} \mathrm{~d} x+c\left(\varepsilon_{1}\right) \int_{0}^{1}\left(3 w_{x}-\psi_{x}\right)^{2} \mathrm{~d} x+c\left(\varepsilon_{1}\right) \int_{0}^{1} w_{x}^{2} \mathrm{~d} x,
$$

for any $\varepsilon_{1}>0$.

Proof. By differentiating $I_{1}(t)$ with respect to $t$, using $(1.5)_{1}$ and integrating by parts, we obtain

$$
I_{1}^{\prime}(t)=-\rho \int_{0}^{1} \varphi_{t}^{2} \mathrm{~d} x-G \int_{0}^{1} \varphi_{x}\left(\psi-\varphi_{x}\right) \mathrm{d} x
$$

Note that

$$
-G \int_{0}^{1}\left(\psi-\varphi_{x}\right) \varphi_{x} \mathrm{~d} x=G \int_{0}^{1}\left(\psi-\varphi_{x}\right)^{2} \mathrm{~d} x-G \int_{0}^{1} \psi\left(\psi-\varphi_{x}\right) \mathrm{d} x .
$$

Then, we deduce that

$$
I_{1}^{\prime}(t)=-\rho \int_{0}^{1} \varphi_{t}^{2} \mathrm{~d} x+G \int_{0}^{1}\left(\psi-\varphi_{x}\right)^{2} \mathrm{~d} x-G \int_{0}^{1} \psi\left(\psi-\varphi_{x}\right) \mathrm{d} x .
$$

Making use of Young's inequality with $\varepsilon_{1}>0$, we obtain

$$
I_{1}^{\prime}(t) \leq-\rho \int_{0}^{1} \varphi_{t}^{2} \mathrm{~d}+\left(G+\varepsilon_{1}\right) \int_{0}^{1}\left(\psi-\varphi_{x}\right)^{2} \mathrm{~d} x+c\left(\varepsilon_{1}\right) \int_{0}^{1} \psi_{x}^{2} \mathrm{~d} x .
$$

Note that

$$
\int_{0}^{1} \psi_{x}^{2} \mathrm{~d} x=\int_{0}^{1}\left(\psi_{x}-3 w_{x}+3 w_{x}\right)^{2} \mathrm{~d} x \leq 2 \int_{0}^{1}\left(3 w_{x}-\psi_{x}\right)^{2} \mathrm{~d} x+18 \int_{0}^{1} w_{x}^{2} \mathrm{~d} x .
$$

Then the estimate (3.9) is established.

Lemma 3.4 Let $(\varphi, \psi, w, \theta)$ be the solution of (1.5)-(1.6). Then the functional

$$
I_{2}(t)=I_{\rho} \int_{0}^{1}(3 w-\psi)(3 w-\psi)_{t} \mathrm{~d} x
$$


satisfies the estimate

$$
\begin{aligned}
I_{2}^{\prime}(t) \leq & -\left(D-\varepsilon_{2}\right) \int_{0}^{1}\left(3 w_{x}-\psi_{x}\right)^{2} \mathrm{~d} x+I_{\rho} \int_{0}^{1}\left(3 w_{t}-\psi_{t}\right)^{2} \mathrm{~d} x \\
& +c\left(\varepsilon_{2}\right) \int_{0}^{1}\left(\psi-\varphi_{x}\right)^{2} \mathrm{~d} x+c\left(\varepsilon_{2}\right) \int_{0}^{1} \theta^{2} \mathrm{~d} x
\end{aligned}
$$

for any $\varepsilon_{2}>0$.

Proof. Taking the derivative of $I_{5}(t)$ with respect to $t$, using $(1.5)_{2}$ and integrating by parts, we get

$$
\begin{aligned}
I_{2}^{\prime}(t)= & -D \int_{0}^{1}\left(3 w_{x}-\psi_{x}\right)^{2} \mathrm{~d} x+I_{\rho} \int_{0}^{1}\left(3 w_{t}-\psi_{t}\right)^{2} \mathrm{~d} x \\
& +G \int_{0}^{1}\left(\psi-\varphi_{x}\right)(3 w-\psi) \mathrm{d} x+\sigma \int_{0}^{1}(3 w-\psi)_{x} \theta \mathrm{d} x .
\end{aligned}
$$

Then, using Young's inequality, we arrive at (3.10).

Lemma 3.5 Let $(\varphi, \psi, w, \theta)$ be the solution of (1.5)-(1.6). Then the functional

$$
I_{3}(t)=I_{\rho} \int_{0}^{1} w w_{t} \mathrm{~d} x
$$

satisfies the estimate

$$
\begin{aligned}
I_{3}^{\prime}(t) \leq & -\left(\frac{4 \gamma}{3}-\varepsilon_{3}\right) \int_{0}^{1} w^{2} \mathrm{~d} x-D \int_{0}^{1} w_{x}^{2} \mathrm{~d} x+\left(I_{\rho}+c\left(\varepsilon_{3}\right)\right) \int_{0}^{1} w_{t}^{2} \mathrm{~d} x \\
& +c\left(\varepsilon_{3}\right) \int_{0}^{1}\left(\psi-\varphi_{x}\right)^{2} \mathrm{~d} x,
\end{aligned}
$$

for any $\varepsilon_{3}>0$.

Proof. By differentiating $I_{1}(t)$ with respect to $t$, using $(1.5)_{3}$ and integrating by parts, we obtain

$$
I_{3}^{\prime}(t)=I_{\rho} \int_{0}^{1} w_{t}^{2} \mathrm{~d} x-G \int_{0}^{1} w\left(\psi-\varphi_{x}\right) \mathrm{d} x-\frac{4 \gamma}{3} \int_{0}^{1} w^{2} \mathrm{~d} x-\frac{4 \beta}{3} \int_{0}^{1} w w_{t} \mathrm{~d} x-D \int_{0}^{1} w_{x}^{2} \mathrm{~d} x .
$$

We then use Young's inequality with $\varepsilon_{3}>0$ to obtain (3.11).

Lemma 3.6 Let $(\varphi, \psi, w, \theta)$ be the solution of (1.5)-(1.6). Then the functional

$$
I_{4}(t)=\frac{k I_{\rho}}{\sigma} \int_{0}^{1}(3 w-\psi)_{t} \int_{0}^{x} \theta \mathrm{d} y \mathrm{~d} x
$$

satisfies the estimate

$$
\begin{aligned}
I_{4}^{\prime}(t) \leq & -\left(I_{\rho}-\varepsilon_{5}\right) \int_{0}^{1}\left(3 w_{t}-\psi_{t}\right)^{2} \mathrm{~d} x+\left(k+c\left(\varepsilon_{4}\right)\right) \int_{0}^{1} \theta^{2} \mathrm{~d} x+\varepsilon_{4} \int_{0}^{1}\left(\psi-\varphi_{x}\right)^{2} \mathrm{~d} x \\
& +\varepsilon_{4} \int_{0}^{1}\left(3 w_{x}-\psi_{x}\right)^{2} \mathrm{~d} x+c\left(\varepsilon_{5}\right) \int_{0}^{1} \theta_{x}^{2} \mathrm{~d} x,
\end{aligned}
$$

for any $\varepsilon_{4}, \varepsilon_{5}>0$. 
Proof. Taking the derivative of $I_{4}(t)$ with respect to $t$, using $(1.5)_{2},(1.5)_{4}$ and integrating by parts, we get

$$
\begin{aligned}
I_{4}^{\prime}(t)= & -I_{\rho} \int_{0}^{1}\left(3 w_{t}-\psi_{t}\right)^{2} \mathrm{~d} x+\frac{k G}{\sigma} \int_{0}^{1}\left(\psi-\varphi_{x}\right) \int_{0}^{x} \theta \mathrm{d} y \mathrm{~d} x-\frac{k D}{\sigma} \int_{0}^{1}(3 w-\psi)_{x} \theta \mathrm{d} x \\
& +k \int_{0}^{1} \theta^{2} \mathrm{~d} x+\frac{\tau I_{\rho}}{\sigma} \int_{0}^{1}(3 w-\psi)_{t} \theta_{x} \mathrm{~d} x .
\end{aligned}
$$

Using Young's inequality with $\varepsilon_{4}, \varepsilon_{5}>0$, we establish the (3.12).

Lemma 3.7 Let $(\varphi, \psi, w, \theta)$ be the solution of (1.5)-(1.6). Then the functional

$$
I_{5}(t)=I_{\rho} \int_{0}^{1} w_{t}\left(\psi-\varphi_{x}\right) \mathrm{d} x+I_{\rho} \int_{0}^{1} w_{t} \varphi_{x} \mathrm{~d} x-\frac{D \rho}{G} \int_{0}^{1}\left(w_{x} \varphi_{t}-w_{x t} \varphi\right) \mathrm{d} x
$$

satisfies the estimate

$$
\begin{aligned}
I_{5}^{\prime}(t) \leq & -\left(G-\varepsilon_{6}\right) \int_{0}^{1}\left(\psi-\varphi_{x}\right)^{2} \mathrm{~d} x+\varepsilon_{6} \int_{0}^{1}\left(3 w_{t}-\psi_{t}\right)^{2} \mathrm{~d} x \\
& +c\left(\varepsilon_{6}\right) \int_{0}^{1} w^{2} \mathrm{~d} x+c\left(\varepsilon_{6}\right) \int_{0}^{1} w_{t}^{2} \mathrm{~d} x
\end{aligned}
$$

for any $\varepsilon_{6}>0$.

Proof. By $(1.5)_{1},(1.5)_{3}$ and integrating by parts, we get

$$
\begin{aligned}
& \frac{d}{d t}\left\{I_{\rho} \int_{0}^{1} w_{t}\left(\psi-\varphi_{x}\right) \mathrm{d} x\right\} \\
= & -D \int_{0}^{1} w_{x}\left(\psi-\varphi_{x}\right)_{x} \mathrm{~d} x-G \int_{0}^{1}\left(\psi-\varphi_{x}\right)^{2} \mathrm{~d} x-\frac{4 \gamma}{3} \int_{0}^{1} w\left(\psi-\varphi_{x}\right) \mathrm{d} x-\frac{4 \beta}{3} \int_{0}^{1} w_{t}\left(\psi-\varphi_{x}\right) \mathrm{d} x \\
& +I_{\rho} \int_{0}^{1} w_{t} \psi_{t} \mathrm{~d} x-I_{\rho} \int_{0}^{1} w_{t} \varphi_{x t} \mathrm{~d} x \\
= & \frac{D \rho}{G} \int_{0}^{1} w_{x} \varphi_{t t} \mathrm{~d} x-G \int_{0}^{1}\left(\psi-\varphi_{x}\right)^{2} \mathrm{~d} x-\frac{4 \gamma}{3} \int_{0}^{1} w\left(\psi-\varphi_{x}\right) \mathrm{d} x-\frac{4 \beta}{3} \int_{0}^{1} w_{t}\left(\psi-\varphi_{x}\right) \mathrm{d} x \\
& +I_{\rho} \int_{0}^{1} w_{t} \psi_{t} \mathrm{~d} x-\frac{d}{d t}\left\{I_{\rho} \int_{0}^{1} w_{t} \varphi_{x} \mathrm{~d} x\right\}+I_{\rho} \int_{0}^{1} w_{t t} \varphi_{x} \mathrm{~d} x \\
= & \frac{D \rho}{G}\left\{\frac{d}{d t} \int_{0}^{1}\left(w_{x} \varphi_{t}-w_{x t} \varphi\right) \mathrm{d} x-\int_{0}^{1} w_{t t} \varphi_{x} \mathrm{~d} x\right\}-G \int_{0}^{1}\left(\psi-\varphi_{x}\right)^{2} \mathrm{~d} x-\frac{4 \gamma}{3} \int_{0}^{1} w\left(\psi-\varphi_{x}\right) \mathrm{d} x \\
& -\frac{4 \beta}{3} \int_{0}^{1} w_{t}\left(\psi-\varphi_{x}\right) \mathrm{d} x+I_{\rho} \int_{0}^{1} w_{t} \psi_{t} \mathrm{~d} x-\frac{d}{d t}\left\{I_{\rho} \int_{0}^{1} w_{t} \varphi_{x} \mathrm{~d} x\right\}+I_{\rho} \int_{0}^{1} w_{t t} \varphi_{x} \mathrm{~d} x .
\end{aligned}
$$

We conclude for

$$
\begin{aligned}
I_{5}^{\prime}(t)= & D\left(\frac{I_{\rho}}{D}-\frac{\rho}{G}\right) \int_{0}^{1} w_{t t} \varphi_{x} \mathrm{~d} x-G \int_{0}^{1}\left(\psi-\varphi_{x}\right)^{2} \mathrm{~d} x-\frac{4 \gamma}{3} \int_{0}^{1} w\left(\psi-\varphi_{x}\right) \mathrm{d} x \\
& -\frac{4 \beta}{3} \int_{0}^{1} w_{t}\left(\psi-\varphi_{x}\right) \mathrm{d} x+I_{\rho} \int_{0}^{1} w_{t} \psi_{t} \mathrm{~d} x .
\end{aligned}
$$

Using Young's inequality and $\frac{\rho}{G}=\frac{I_{\rho}}{D}$, we get (3.13). 
Now, we turn to prove our main result in this section.

Proof of Theorem 3.1. Let $\delta_{1}, \delta_{2}, \delta_{3}, \delta_{4}, \delta_{5}>0$ and $\frac{\rho}{G}=\frac{I_{\rho}}{D}$, we define

$$
L_{1}(t)=E(t)+\delta_{1} I_{1}(t)+\delta_{2} I_{2}(t)+\delta_{3} I_{3}(t)+\delta_{4} I_{4}(t)+\delta_{5} I_{5}(t) .
$$

Using Cauchy-Schwarz inequality and Poincaré inequality, one can easily see that all the $I_{i}(t), i=$ $1,2,3,4,5$ are bounded by an expression containing the existing terms in the energy $E(t)$. This leads to the equivalence of $L_{1}(t)$ and $E(t)$.

Gathering the estimates in the previous lemmas, we obtain

$$
\begin{aligned}
L_{1}^{\prime}(t) \leq & -\delta_{1} \rho \int_{0}^{1} \varphi_{t}^{2} \mathrm{~d} x-\left(\delta_{4} I_{\rho}-\delta_{4} \varepsilon_{5}-\delta_{2} I_{\rho}-\delta_{5} \varepsilon_{6}\right) \int_{0}^{1}\left(3 w_{t}-\psi_{t}\right)^{2} \mathrm{~d} x \\
& -\left(4 \beta-\delta_{3} I_{\rho}-\delta_{3} c\left(\varepsilon_{3}\right)-\delta_{5} c\left(\varepsilon_{6}\right)\right) \int_{0}^{1} w_{t}^{2} \mathrm{~d} x \\
& -\left(\delta_{5} G-\delta_{5} \varepsilon_{6}-\delta_{1} G-\delta_{1} \varepsilon_{1}-\delta_{2} c\left(\varepsilon_{2}\right)-\delta_{3} c\left(\varepsilon_{3}\right)-\delta_{4} \varepsilon_{4}\right) \int_{0}^{1}\left(\psi-\varphi_{x}\right)^{2} \mathrm{~d} x \\
& -\left(\delta_{2} D-\delta_{1} c\left(\varepsilon_{1}\right)-\delta_{2} \varepsilon_{2}-\delta_{4} \varepsilon_{4}\right) \int_{0}^{1}\left(3 w_{x}-\psi_{x}\right)^{2} \mathrm{~d} x \\
& -\left(\delta_{3} D-\delta_{1} c\left(\varepsilon_{1}\right)\right) \int_{0}^{1} w_{x}^{2} \mathrm{~d} x-\left(\frac{4 \gamma}{3} \delta_{3}-\delta_{3} \varepsilon_{3}-\delta_{5} c\left(\varepsilon_{6}\right)\right) \int_{0}^{1} w^{2} \mathrm{~d} x \\
& -\left(\tau-\delta_{4} c\left(\varepsilon_{5}\right)\right) \int_{0}^{1} \theta_{x}^{2} \mathrm{~d} x+\left(\delta_{2} c\left(\varepsilon_{2}\right)+\delta_{4} k+\delta_{4} c\left(\varepsilon_{4}\right)\right) \int_{0}^{1} \theta^{2} \mathrm{~d} x
\end{aligned}
$$

At this point, we need to choose our constants very carefully. First, we choose $\varepsilon_{1}, \varepsilon_{2}, \varepsilon_{3}, \varepsilon_{4}, \varepsilon_{5}, \varepsilon_{6}$ small enough so that

$$
\begin{aligned}
L_{1}^{\prime}(t) \leq & -\delta_{1} \rho \int_{0}^{1} \varphi_{t}^{2} \mathrm{~d} x-\left(\frac{\delta_{4} I_{\rho}}{2}-\delta_{2} I_{\rho}\right) \int_{0}^{1}\left(3 w_{t}-\psi_{t}\right)^{2} \mathrm{~d} x \\
& -\left(4 \beta-\delta_{3} I_{\rho}-\delta_{3} c\left(\varepsilon_{3}\right)-\delta_{5} c\left(\varepsilon_{6}\right)\right) \int_{0}^{1} w_{t}^{2} \mathrm{~d} x \\
& -\left(\frac{\delta_{5} G}{2}-\delta_{1} G-\delta_{2} c\left(\varepsilon_{2}\right)-\delta_{3} c\left(\varepsilon_{3}\right)\right) \int_{0}^{1}\left(\psi-\varphi_{x}\right)^{2} \mathrm{~d} x \\
& -\left(\frac{\delta_{2} D}{2}-\delta_{1} c\left(\varepsilon_{1}\right)\right) \int_{0}^{1}\left(3 w_{x}-\psi_{x}\right)^{2} \mathrm{~d} x-\left(\delta_{3} D-\delta_{1} c\left(\varepsilon_{1}\right)\right) \int_{0}^{1} w_{x}^{2} \mathrm{~d} x \\
& -\left(\frac{2 \gamma}{3} \delta_{3}-\delta_{5} c\left(\varepsilon_{6}\right)\right) \int_{0}^{1} w^{2} \mathrm{~d} x-\left(\tau-\delta_{4} c\left(\varepsilon_{5}\right)\right) \int_{0}^{1} \theta_{x}^{2} \mathrm{~d} x \\
& +\left(\delta_{2} c\left(\varepsilon_{2}\right)+\delta_{4} k+\delta_{4} c\left(\varepsilon_{4}\right)\right) \int_{0}^{1} \theta^{2} \mathrm{~d} x .
\end{aligned}
$$

Then, we select $\delta_{4}$ small enough so that

$$
\tau-\delta_{4} c\left(\varepsilon_{5}\right)>0
$$

Next, we choose $\delta_{2}$ small enough so that

$$
\frac{\delta_{4} I_{\rho}}{2}-\delta_{2} I_{\rho}>0
$$


Furthermore, we select $\delta_{3}$ and $\delta_{5}$ small enough so that

$$
4 \beta-\delta_{3} I_{\rho}-\delta_{3} c\left(\varepsilon_{3}\right)-\delta_{5} c\left(\varepsilon_{6}\right)>0, \delta_{3} D-\delta_{1} c\left(\varepsilon_{1}\right)>0, \frac{\delta_{5} G}{2}-\delta_{3} c\left(\varepsilon_{3}\right)>0 .
$$

Finally, we select $\delta_{3}$ even smaller (if needed) and $\delta_{1}$ small enough so that

$$
\frac{\delta_{2} D}{2}-\delta_{1} c\left(\varepsilon_{1}\right)>0, \delta_{3} D-\delta_{1} c\left(\varepsilon_{1}\right)>0, \frac{\delta_{5} G}{2}-\delta_{1} G-\delta_{2} c\left(\varepsilon_{2}\right)-\delta_{3} c\left(\varepsilon_{3}\right)>0 .
$$

From the above, we deduce that there exist positive constants $C_{1}$ and $C_{2}$ such that (3.16) becomes

$$
\begin{aligned}
L_{1}^{\prime}(t) & \leq-C_{1} E(t)-\left(\tau-\delta_{4} c\left(\varepsilon_{5}\right)\right) \int_{0}^{1} \theta_{x}^{2} \mathrm{~d} x+C_{2} \int_{0}^{1} \theta^{2} \mathrm{~d} x \\
& \leq-C_{1} E(t)+C_{2} \int_{0}^{1} \theta_{x}^{2} \mathrm{~d} x
\end{aligned}
$$

By (3.3), we get

$$
L_{1}^{\prime}(t) \leq-C_{1} E(t)-C_{3} E^{\prime}(t)
$$

for some positive constant $C_{3}$. It is obvious that

$$
\mathscr{L}_{1}(t)=L(t)+C_{3} E(t) \sim E(t) .
$$

Recalling (3.18), we obtain

$$
\mathscr{L}_{1}^{\prime}(t)=L^{\prime}(t)+C_{3} E^{\prime}(t) \leq-C_{1} E(t) \leq-c \mathscr{L}_{1}(t),
$$

for some positive constant $c_{1}$. Then, a simple integration of $(3.19)$ over $(0, t)$ yields

$$
\mathscr{L}_{1}(t) \leq \mathscr{L}_{1}(0) e^{-c_{1} t}, \forall t \geq 0
$$

At last, estimate (3.20) gives the desired result (3.2) when combined with the equivalence of $L(t)$ and $E(t)$.

\section{The lack of exponential stability}

This Section is concerning the lack of exponential stability. Our result is achieved by GearhartHerbst-Prüss-Huang theorem to dissipative systems, see Prüss [21] and Huang [22].

Theorem 4.1 Let $S(t)=e^{\mathcal{A t}}$ be a $C_{0}$-semigroup of contractions on Hilbert space $\mathcal{H}$. Then $S(t)$ is exponentially stable if and only if

$$
\rho(\mathcal{A}) \supset\{i \lambda: \lambda \in \mathbb{R}\} \equiv i \mathbb{R}
$$

and

$$
\varlimsup_{|\lambda| \rightarrow \infty}\left\|(i \lambda I-\mathcal{A})^{-1}\right\|_{\mathcal{L}(\mathcal{H})}<\infty
$$

hold, where $\rho(\mathcal{A})$ is the resolvent set of the differential operator $\mathcal{A}$.

Next, we state and prove the main result of this section. 
Theorem 4.2 Assume that $\frac{\rho}{G} \neq \frac{I_{\rho}}{D}$ hold. Then the semigroup associated to problem (1.5)-(1.6) is not exponentially stable.

Proof. We will prove that there exists a sequence of imaginary number $\lambda_{\mu}$ and function $F_{\mu} \in \mathcal{H}$ with $\left\|F_{\mu}\right\|_{\mathcal{H}} \leq 1$ such that $\left\|\left(\lambda_{\mu} I-\mathcal{A}\right)^{-1} F_{\mu}\right\|_{\mathcal{H}}=\left\|U_{\mu}\right\|_{\mathcal{H}} \rightarrow \infty$, where

$$
\lambda_{\mu} U_{\mu}-\mathcal{A} U_{\mu}=F_{\mu},
$$

with $U_{\mu}=\left(v_{1}, v_{2}, v_{3}, v_{4}, v_{5}, v_{6}, v_{7}\right)^{T}$ not bounded. Rewrite spectral equation (4.1) in term of its components, we have for $\lambda_{\mu}=\lambda$

$$
\left\{\begin{array}{l}
\lambda v_{1}-v_{2}=g_{1} \\
\rho \lambda v_{2}-G \partial_{x x} v_{1}-G \partial_{x} v_{3}+3 G \partial_{x} v_{5}=\rho g_{2} \\
\lambda v_{3}-v_{4}=g_{3} \\
I_{\rho} \lambda v_{4}+G \partial_{x} v_{1}+G v_{3}-D \partial_{x x} v_{3}-3 G v_{5}+\sigma \partial_{x} v_{7}=I_{\rho} g_{4} \\
\lambda v_{5}-v_{6}=g_{5} \\
I_{\rho} \lambda v_{6}+\frac{4 \beta}{3} v_{6}-G \partial_{x} v_{1}-G v_{3}+\left(3 G+\frac{4 \gamma}{3}\right) v_{5}-D \partial_{x x} v_{5}=I_{\rho} g_{6} \\
k \lambda v_{7}-\tau \partial_{x x} v_{7}+\sigma \partial_{x} v_{4}=k g_{7}
\end{array}\right.
$$

where $\lambda \in \mathbb{R}$ and $F=\left(g_{1}, g_{2}, g_{3}, g_{4}, g_{5}, g_{6}, g_{7}\right)^{T} \in \mathcal{H}$. Taking $g_{1}=g_{3}=g_{5}=0$, then the above system becomes

$$
\left\{\begin{array}{l}
\rho \lambda^{2} v_{1}-G \partial_{x x} v_{1}-G \partial_{x} v_{3}+3 G \partial_{x} v_{5}=\rho g_{2} \\
I_{\rho} \lambda^{2} v_{3}+G \partial_{x} v_{1}+G v_{3}-D \partial_{x x} v_{3}-3 G v_{5}+\sigma \partial_{x} v_{7}=I_{\rho} g_{4}, \\
I_{\rho} \lambda^{2} v_{5}+\frac{4 \beta}{3} \lambda v_{5}-G \partial_{x} v_{1}-G v_{3}+\left(3 G+\frac{4 \gamma}{3}\right) v_{5}-D \partial_{x x} v_{5}=I_{\rho} g_{6} \\
k \lambda v_{7}-\tau \partial_{x x} v_{7}+\lambda \sigma \partial_{x} v_{3}=k g_{7} .
\end{array}\right.
$$

Because of the boundary conditions in (1.6), we can suppose that

$$
v_{1}=A \sin \left(\frac{\mu \pi}{2} x\right), v_{3}=B \cos \left(\frac{\mu \pi}{2} x\right), v_{5}=C \cos \left(\frac{\mu \pi}{2} x\right), v_{7}=E \sin \left(\frac{\mu \pi}{2} x\right) .
$$

Now, choosing

$$
g_{2}=\frac{1}{\rho} \sin \left(\frac{\mu \pi}{2} x\right), g_{4}=g_{6}=g_{7}=0
$$

we arrive at

$$
\left\{\begin{array}{l}
\left(\rho \lambda^{2}+G\left(\frac{\mu \pi}{2}\right)^{2}\right) A+G\left(\frac{\mu \pi}{2}\right) B-3 G\left(\frac{\mu \pi}{2}\right) C=1 \\
G\left(\frac{\mu \pi}{2}\right) A+\left(I_{\rho} \lambda^{2}+G+D\left(\frac{\mu \pi}{2}\right)^{2}\right) B-3 G C+\sigma\left(\frac{\mu \pi}{2}\right) E=0 \\
-G\left(\frac{\mu \pi}{2}\right) A-G B+\left(I_{\rho} \lambda^{2}+\frac{4 \beta}{3} \lambda+3 G+\frac{4 \gamma}{3}+D\left(\frac{\mu \pi}{2}\right)^{2}\right) C=0 \\
-\lambda \sigma\left(\frac{\mu \pi}{2}\right) B+\left(k \lambda+\tau\left(\frac{\mu \pi}{2}\right)^{2}\right) E=0 .
\end{array}\right.
$$


Now, we take $\lambda=\lambda_{\mu}$ such that

$$
\rho \lambda^{2}+G\left(\frac{\mu \pi}{2}\right)^{2}=0,
$$

then the above system can be written as

$$
\left\{\begin{array}{l}
G\left(\frac{\mu \pi}{2}\right) B-3 G\left(\frac{\mu \pi}{2}\right) C=1 \\
G\left(\frac{\mu \pi}{2}\right) A+\left(G+I_{\rho}\left(\frac{D}{I_{\rho}}-\frac{G}{\rho}\right)\left(\frac{\mu \pi}{2}\right)^{2}\right) B-3 G C+\sigma\left(\frac{\mu \pi}{2}\right) E=0 \\
-G\left(\frac{\mu \pi}{2}\right) A-G B+\left(\frac{4 \beta}{3} \lambda+3 G+\frac{4 \gamma}{3}+I_{\rho}\left(\frac{D}{I_{\rho}}-\frac{G}{\rho}\right)\left(\frac{\mu \pi}{2}\right)^{2}\right) C=0 \\
-i \sigma \sqrt{\frac{G}{\rho}}\left(\frac{\mu \pi}{2}\right)^{2} B+\left(i k \sqrt{\frac{G}{\rho}}\left(\frac{\mu \pi}{2}\right)+\tau\left(\frac{\mu \pi}{2}\right)^{2}\right) E=0
\end{array}\right.
$$

Adding $(4.5)_{2}$ to $(4.5)_{3}$, we get

$$
I_{\rho}\left(\frac{D}{I_{\rho}}-\frac{G}{\rho}\right)\left(\frac{\mu \pi}{2}\right)^{2} B+\sigma\left(\frac{\mu \pi}{2}\right) E+\left(\frac{4 \beta}{3} \lambda+\frac{4 \gamma}{3}+I_{\rho}\left(\frac{D}{I_{\rho}}-\frac{G}{\rho}\right)\left(\frac{\mu \pi}{2}\right)^{2}\right) C=0 .
$$

From $(4.5)_{4}$, we get

$$
E=\frac{i \sigma \sqrt{\frac{G}{\rho}}\left(\frac{\mu \pi}{2}\right)}{i k \sqrt{\frac{G}{\rho}}+\tau\left(\frac{\mu \pi}{2}\right)} B
$$

Substituting $E$ into (4.6), we get

$$
C=-\frac{\Lambda_{\mu}}{\Gamma_{\mu}} B
$$

where

$$
\begin{gathered}
\Lambda_{\mu}=I_{\rho}\left(\frac{D}{I_{\rho}}-\frac{G}{\rho}\right)\left(\frac{\mu \pi}{2}\right)^{2}+\frac{i \sigma^{2} \sqrt{\frac{G}{\rho}}\left(\frac{\mu \pi}{2}\right)^{2}}{i k \sqrt{\frac{G}{\rho}}+\tau\left(\frac{\mu \pi}{2}\right)}, \\
\Gamma_{\mu}=\frac{4 \beta}{3} \lambda+\frac{4 \gamma}{3}+I_{\rho}\left(\frac{D}{I_{\rho}}-\frac{G}{\rho}\right)\left(\frac{\mu \pi}{2}\right)^{2} .
\end{gathered}
$$

Substituting $C$ into $(4.5)_{3}$, we get

$$
A=-\frac{G \Gamma_{\mu}+\Lambda_{\mu} \Gamma_{\mu}+3 G \Lambda_{\mu}}{G\left(\frac{\mu \pi}{2}\right) \Gamma_{\mu}} B
$$

Similarly, substituting $C$ into $(4.5)_{1}$, we get

$$
B=\frac{\Gamma_{\mu}}{G\left(\frac{\mu \pi}{2}\right)\left(\Gamma_{\mu}+3 \Lambda_{\mu}\right)} .
$$

Let $\mu \rightarrow \infty$, we get

$$
\left(\frac{\mu \pi}{2}\right) B \rightarrow \frac{1}{4 G}
$$

Substituting this expression into $A, C$ and $E$, we obtain for $\mu \rightarrow \infty$,

$$
A \rightarrow \frac{D}{4 \rho G}\left(\frac{\rho}{G}-\frac{I_{\rho}}{D}\right), C \rightarrow O\left(\frac{1}{\mu}\right), E \rightarrow O\left(\frac{1}{\mu}\right) .
$$


Thus

$$
\begin{aligned}
\left\|U_{\mu}\right\|_{\mathcal{H}}^{2} & \geq G \int_{0}^{1}\left(\psi-\varphi_{x}\right)^{2} \mathrm{~d} x=G\left(3 C-B-\left(\frac{\mu \pi}{2}\right) A\right)^{2} \int_{0}^{1} \cos ^{2}\left(\frac{\mu \pi}{2} x\right) \mathrm{d} x \\
& =\frac{1}{2} G\left(3 C-B-\left(\frac{\mu \pi}{2}\right) A\right)^{2} \rightarrow \infty, \text { as } \mu \rightarrow \infty .
\end{aligned}
$$

Therefore, there is no exponential stability. This completes the proof.

\section{Polynomial stability}

In this section, we consider the situation when the wave propagations are not the same.

Theorem 5.1 Assume that $\frac{\rho}{G} \neq \frac{I_{\rho}}{D}$ hold. Let $U^{0} \in \mathscr{H}$, then there exists a positive constant $c_{2}$ such that the energy $E(t)$ associated with problem (1.5)-(1.6) satisfies

$$
E(t) \leq \frac{c_{2}}{t}, t>0 \text {. }
$$

Proof. In this regard, we establish a polynomial decay result. As we will see, due to the presence of the $\int_{0}^{1} w_{t t} \varphi_{x} \mathrm{~d} x$, we cannot directly perform the same proof as for the case where $\frac{\rho}{G} \neq \frac{I_{\rho}}{D}$. To overcome this difficulty, the second-order energy method is needed. The second-order energy is defined by

$$
\begin{aligned}
\mathcal{E}(t)= & \frac{1}{2}\left(\rho \int_{0}^{1} \varphi_{t t}^{2} \mathrm{~d} x+I_{\rho} \int_{0}^{1}\left(3 w_{t t}-\psi_{t t}\right)^{2} \mathrm{~d} x+3 I_{\rho} \int_{0}^{1} w_{t t}^{2} \mathrm{~d} x+G \int_{0}^{1}\left(\psi_{t}-\varphi_{x t}\right)^{2} \mathrm{~d} x\right. \\
& \left.+D \int_{0}^{1}\left(3 w_{x t}-\psi_{x t}\right)^{2} \mathrm{~d} x+3 D \int_{0}^{1} w_{x t}^{2} \mathrm{~d} x+4 \gamma \int_{0}^{1} w_{t}^{2} \mathrm{~d} x+k \int_{0}^{1} \theta_{t}^{2} \mathrm{~d} x\right) .
\end{aligned}
$$

A simple calculation (Similar to (3.3)) implies that

$$
\mathcal{E}^{\prime}(t)=-4 \beta \int_{0}^{1} w_{t t}^{2} \mathrm{~d} x-\tau \int_{0}^{1} \theta_{x t}^{2} \mathrm{~d} x .
$$

As in (3.14), we also define a Lyapunov functional $L_{2}(t)$ as follows:

$$
L_{2}(t)=E(t)+\mathcal{E}(t)+\delta_{1} I_{1}(t)+\delta_{2} I_{2}(t)+\delta_{3} I_{3}(t)+\delta_{4} I_{4}(t)+\delta_{5} I_{5}(t),
$$

where $I_{i}(t), i=1,2,3,4$ remain as defined in Lemma 3.3-Lemma 3.4 with derivatives of $I_{1}(t)-I_{4}(t)$ remain the same while the derivative of $I_{5}(t)$ is given as

$$
\begin{aligned}
I_{5}^{\prime}(t)= & D\left(\frac{I_{\rho}}{D}-\frac{\rho}{G}\right) \int_{0}^{1} w_{t t} \varphi_{x} \mathrm{~d} x-G \int_{0}^{1}\left(\psi-\varphi_{x}\right)^{2} \mathrm{~d} x-\frac{4 \gamma}{3} \int_{0}^{1} w\left(\psi-\varphi_{x}\right) \mathrm{d} x \\
& -\frac{4 \beta}{3} \int_{0}^{1} w_{t}\left(\psi-\varphi_{x}\right) \mathrm{d} x+\int_{0}^{1} I_{\rho} w_{t} \psi_{t} \mathrm{~d} x \\
\leq & -\left(G-\varepsilon_{6}\right) \int_{0}^{1}\left(\psi-\varphi_{x}\right)^{2} \mathrm{~d} x+\varepsilon_{6} \int_{0}^{1}\left(3 w_{t}-\psi_{t}\right)^{2} \mathrm{~d} x+c\left(\varepsilon_{6}\right) \int_{0}^{1} w^{2} \mathrm{~d} x \\
& +c\left(\varepsilon_{6}\right) \int_{0}^{1} w_{t}^{2} \mathrm{~d} x+c\left(\varepsilon_{7}\right) \int_{0}^{1} w_{t t}^{2} \mathrm{~d} x+\varepsilon_{7} \int_{0}^{1} \varphi_{x}^{2} \mathrm{~d} x
\end{aligned}
$$


for any $\varepsilon_{6}, \varepsilon_{7}>0$. Observing

$$
\begin{aligned}
\int_{0}^{1} \varphi_{x}^{2} \mathrm{~d} x & \leq 2 \int_{0}^{1}\left(\psi-\varphi_{x}\right)^{2} \mathrm{~d} x+2 \int_{0}^{1} \psi^{2} \mathrm{~d} x \\
& \leq 2 \int_{0}^{1}\left(\psi-\varphi_{x}\right)^{2} \mathrm{~d} x+4 \int_{0}^{1}\left(3 w_{x}-\psi_{x}\right)^{2} \mathrm{~d} x+36 \int_{0}^{1} w_{x}^{2} \mathrm{~d} x .
\end{aligned}
$$

Then, combining (5.4)-(5.5), we get

$$
\begin{aligned}
I_{5}^{\prime}(t) \leq & -\left(G-\varepsilon_{6}-\varepsilon_{7}\right) \int_{0}^{1}\left(\psi-\varphi_{x}\right)^{2} \mathrm{~d} x+\varepsilon_{6} \int_{0}^{1}\left(3 w_{t}-\psi_{t}\right)^{2} \mathrm{~d} x+c\left(\varepsilon_{6}\right) \int_{0}^{1} w^{2} \mathrm{~d} x \\
& +c\left(\varepsilon_{6}\right) \int_{0}^{1} w_{t}^{2} \mathrm{~d} x+c\left(\varepsilon_{7}\right) \int_{0}^{1} w_{t t}^{2} \mathrm{~d} x+\varepsilon_{7} \int_{0}^{1}\left(3 w_{x}-\psi_{x}\right)^{2} \mathrm{~d} x+\varepsilon_{7} \int_{0}^{1} w_{x}^{2} \mathrm{~d} x .
\end{aligned}
$$

Next, differentiating $L_{2}(t)$, we obtain

$$
\begin{aligned}
L_{2}^{\prime}(t) \leq & -\delta_{1} \rho \int_{0}^{1} \varphi_{t}^{2} \mathrm{~d} x-\left(\delta_{4} I_{\rho}-\delta_{4} \varepsilon_{5}-\delta_{2} I_{\rho}-\delta_{5} \varepsilon_{6}\right) \int_{0}^{1}\left(3 w_{t}-\psi_{t}\right)^{2} \mathrm{~d} x \\
& -\left(4 \beta-\delta_{3} I_{\rho}-\delta_{3} c\left(\varepsilon_{3}\right)-\delta_{5} c\left(\varepsilon_{6}\right)\right) \int_{0}^{1} w_{t}^{2} \mathrm{~d} x \\
& -\left(\delta_{5} G-\delta_{5} \varepsilon_{6}-\delta_{1} G-\delta_{1} \varepsilon_{1}-\delta_{2} c\left(\varepsilon_{2}\right)-\delta_{3} c\left(\varepsilon_{3}\right)-\delta_{4} \varepsilon_{4}-\delta_{5} \varepsilon_{7}\right) \int_{0}^{1}\left(\psi-\varphi_{x}\right)^{2} \mathrm{~d} x \\
& -\left(\delta_{2} D-\delta_{1} c\left(\varepsilon_{1}\right)-\delta_{2} \varepsilon_{2}-\delta_{4} \varepsilon_{4}-\delta_{5} \varepsilon_{7}\right) \int_{0}^{1}\left(3 w_{x}-\psi_{x}\right)^{2} \mathrm{~d} x \\
& -\left(\delta_{3} D-\delta_{1} c\left(\varepsilon_{1}\right)-\delta_{5} \varepsilon_{7}\right) \int_{0}^{1} w_{x}^{2} \mathrm{~d} x-\left(\frac{4 \gamma}{3} \delta_{3}-\delta_{3} \varepsilon_{3}-\delta_{5} c\left(\varepsilon_{6}\right)\right) \int_{0}^{1} w^{2} \mathrm{~d} x \\
& -\left(\tau-\delta_{4} c\left(\varepsilon_{5}\right)\right) \int_{0}^{1} \theta_{x}^{2} \mathrm{~d} x+\left(\delta_{2} c\left(\varepsilon_{2}\right)+\delta_{4} k+\delta_{4} c\left(\varepsilon_{4}\right)\right) \int_{0}^{1} \theta^{2} \mathrm{~d} x+\delta_{5} c\left(\varepsilon_{7}\right) \int_{0}^{1} w_{t t}^{2} \mathrm{~d} x .
\end{aligned}
$$

At this point, we need to choose our constants very carefully. First, we choose $\varepsilon_{1}, \varepsilon_{2}, \varepsilon_{3}, \varepsilon_{4}, \varepsilon_{5}$ small enough so that

$$
\begin{aligned}
L_{2}^{\prime}(t) \leq & -\delta_{1} \rho \int_{0}^{1} \varphi_{t}^{2} \mathrm{~d} x-\left(\frac{\delta_{4} I_{\rho}}{2}-\delta_{2} I_{\rho}\right) \int_{0}^{1}\left(3 w_{t}-\psi_{t}\right)^{2} \mathrm{~d} x \\
& -\left(4 \beta-\delta_{3} I_{\rho}-\delta_{3} c\left(\varepsilon_{3}\right)-\delta_{5} c\left(\varepsilon_{6}\right)\right) \int_{0}^{1} w_{t}^{2} \mathrm{~d} x \\
& -\left(\frac{\delta_{5} G}{2}-\delta_{1} G--\delta_{2} c\left(\varepsilon_{2}\right)-\delta_{3} c\left(\varepsilon_{3}\right)\right) \int_{0}^{1}\left(\psi-\varphi_{x}\right)^{2} \mathrm{~d} x \\
& -\left(\frac{\delta_{2} D}{2}-\delta_{1} c\left(\varepsilon_{1}\right)\right) \int_{0}^{1}\left(3 w_{x}-\psi_{x}\right)^{2} \mathrm{~d} x-\left(\frac{\delta_{3} D}{2}-\delta_{1} c\left(\varepsilon_{1}\right)\right) \int_{0}^{1} w_{x}^{2} \mathrm{~d} x \\
& -\left(\frac{2 \gamma}{3} \delta_{3}-\delta_{5} c\left(\varepsilon_{6}\right)\right) \int_{0}^{1} w^{2} \mathrm{~d} x-\left(\tau-\delta_{4} c\left(\varepsilon_{5}\right)\right) \int_{0}^{1} \theta_{x}^{2} \mathrm{~d} x \\
& +\left(\delta_{2} c\left(\varepsilon_{2}\right)+\delta_{4} k+\delta_{4} c\left(\varepsilon_{4}\right)\right) \int_{0}^{1} \theta^{2} \mathrm{~d} x+\delta_{5} c\left(\varepsilon_{7}\right) \int_{0}^{1} w_{t t}^{2} \mathrm{~d} x
\end{aligned}
$$

Then, we select $\delta_{4}$ small enough so that

$$
\tau-\delta_{4} c\left(\varepsilon_{5}\right)>0 .
$$


Next, we choose $\delta_{2}$ small enough so that

$$
\frac{\delta_{4} I_{\rho}}{2}-\delta_{2} I_{\rho}>0
$$

Furthermore, we select $\delta_{3}$ and $\delta_{5}$ small enough so that

$$
4 \beta-\delta_{3} I_{\rho}-\delta_{3} c\left(\varepsilon_{3}\right)-\delta_{5} c\left(\varepsilon_{6}\right)>0, \delta_{3} D-\delta_{1} c\left(\varepsilon_{1}\right)>0, \frac{\delta_{5} G}{2}-\delta_{3} c\left(\varepsilon_{3}\right)>0 .
$$

Finally, we select $\delta_{3}$ even smaller (if needed) and $\delta_{1}$ small enough so that

$$
\frac{\delta_{2} D}{2}-\delta_{1} c\left(\varepsilon_{1}\right)>0, \delta_{3} D-\delta_{1} c\left(\varepsilon_{1}\right)>0, \frac{\delta_{5} G}{2}-\delta_{1} G-\delta_{2} c\left(\varepsilon_{2}\right)-\delta_{3} c\left(\varepsilon_{3}\right)>0 .
$$

Thus, we deduce that there exist positive constants $C_{4}, C_{5}$ and $C_{6}$ such that (5.8) becomes

$$
\begin{aligned}
L_{2}^{\prime}(t) & \leq-C_{4} E(t)-\left(\tau-\delta_{4} c\left(\varepsilon_{5}\right)\right) \int_{0}^{1} \theta_{x}^{2} \mathrm{~d} x+C_{5} \int_{0}^{1} \theta^{2} \mathrm{~d} x+C_{6} \int_{0}^{1} w_{t t}^{2} \mathrm{~d} x \\
& \leq-C_{4} E(t)+C_{5} \int_{0}^{1} \theta_{x}^{2} \mathrm{~d} x+C_{6} \int_{0}^{1} w_{t t}^{2} \mathrm{~d} x .
\end{aligned}
$$

By (3.3) and (5.2), we get

$$
L_{2}^{\prime}(t) \leq-C_{4} E(t)-C_{7} E^{\prime}(t)-C_{8} \mathcal{E}^{\prime}(t),
$$

for some positive constant $C_{7}$ and $C_{8}$. It is obvious that

$$
\mathscr{L}_{2}(t)=L_{2}(t)+C_{7} E(t)+C_{8} \mathcal{E}(t) \sim L_{2}(t) .
$$

Next, recalling (5.10), we obtain

$$
\mathscr{L}_{2}^{\prime}(t)=L_{2}^{\prime}(t)+C_{7} E^{\prime}(t)+C_{8} \mathcal{E}^{\prime}(t) \leq-C_{4} E(t) .
$$

From the above, we deduce that there exist positive constant $c$ such that (5.12) becomes

$$
L_{2}^{\prime}(t) \leq-c E(t) .
$$

A simple integration of $(5.13)$ over $(0, t)$, recalling that $E$ is non-increasing, yields

$$
t E(t) \leq \int_{0}^{t} E(s) \mathrm{d} s \leq \frac{1}{c}\left(L_{2}(0)-L_{2}(t)\right) \leq \frac{L_{2}(0)}{c} .
$$

Finally, for a positive constant $c_{2}$, we have

$$
E(t) \leq \frac{c_{2}}{t}, \forall t>0
$$

which completes the proof.

\section{Acknowledgments}

This work was supported by the National Natural Science Foundation of China [grant number 11301277], the Natural Science Foundation of Jiangsu Province [grant number BK20151523], the Six Talent Peaks Project in Jiangsu Province [grant number 2015-XCL-020] and the Qing Lan Project of Jiangsu Province. 


\section{References}

[1] J.-M. Wang, G.-Q. Xu and S.-P. Yung, Exponential stabilization of laminated beams with structural damping and boundary feedback controls, SIAM J. Control Optim. 44 (2005), no. $5,1575-1597$.

[2] S. W. Hansen and R. Spies, Structural damping in a laminated beams due to interfacial slip, J. Sound Vibration. 204 (1997), no. 2, 183-202.

[3] X.-G. Cao, D.-Y. Liu and G.-Q. Xu, Easy test for stability of laminated beams with structural damping and boundary feedback controls, J. Dyn. Control Syst. 13 (2007), no. 3, 313-336.

[4] C. A. Raposo, Exponential stability for a structure with interfacial slip and frictional damping, Appl. Math. Lett. 53 (2016), 85-91.

[5] J. E. Muñoz Rivera and R. Racke, Mildly dissipative nonlinear Timoshenko systems - global existence and exponential stability, J. Math. Anal. Appl. 276 (2002), no. 1, 248-278.

[6] D. S. Almeida Júnior, M. L. Santos and J. E. Muñoz Rivera, Stability to 1-D thermoelastic Timoshenko beam acting on shear force, Z. Angew. Math. Phys. 65 (2014), no. 6, 1233-1249.

[7] J. Bajkowski et al., A thermoviscoelastic beam model for brakes, European J. Appl. Math. 15 (2004), no. 2, 181-202.

[8] F. Boulanouar and S. Drabla, General boundary stabilization result of memory-type thermoelasticity with second sound, Electron. J. Differential Equations 2014 (2014), no. 202, 18 pp.

[9] A. D. S. Campelo, D. S. Almeida Júnior and M. L. Santos, Stability to the dissipative Reissner-Mindlin-Timoshenko acting on displacement equation, European J. Appl. Math. 27 (2016), no. 2, 157-193.

[10] M. M. Cavalcanti et al., Uniform decay rates for the energy of Timoshenko system with the arbitrary speeds of propagation and localized nonlinear damping, Z. Angew. Math. Phys. 65 (2014), no. 6, 1189-1206.

[11] M. M. Chen, W. J. Liu and W. C. Zhou, Existence and general stabilization of the Timoshenko system of thermo-viscoelasticity of type III with frictional damping and delay terms, Adv. Nonlinear Anal., in press. doi:10.1515/anona-2016-0085

[12] A. A. Keddi, T. A. Apalaras and S. A. Messaoudi, Exponential and polynomial decay in a thermoelastic-Bresse system with second sound, Appl. Math. Optim., in press. DOI: 10.1007/s00245-016-9376-y

[13] W. J. Liu, K. W. Chen and J. Yu, Existence and general decay for the full von Kármán beam with a thermo-viscoelastic damping, frictional dampings and a delay term, IMA Journal of Mathematical Control and Information, in press. doi: 10.1093/imamci/dnv056

[14] W. J. Liu, K. W. Chen and J. Yu, Asymptotic stability for a non-autonomous full von Kármán beam with thermo-viscoelastic damping, Appl. Anal., in press. doi: 10.1080/00036811.2016.1268688 
[15] S. A. Messaoudi and A. Fareh, Energy decay in a Timoshenko-type system of thermoelasticity of type III with different wave-propagation speeds, Arab. J. Math. (Springer) 2 (2013), no. 2, 199-207.

[16] Y. Qin, X.-G. Yang and Z. Ma, Global existence of solutions for the thermoelastic Bresse system, Commun. Pure Appl. Anal. 13 (2014), no. 4, 1395-1406.

[17] F. Tahamtani and A. Peyravi, Asymptotic behavior and blow-up of solution for a nonlinear viscoelastic wave equation with boundary dissipation, Taiwanese J. Math. 17 (2013), no. 6, 1921-1943.

[18] A. Pazy, Semigroups of linear operators and applications to partial differential equations, Applied Mathematical Sciences, 44, Springer, New York, 1983.

[19] H. Brezis, Functional analysis, Sobolev spaces and partial differential equations, Universitext, Springer, New York, 2011.

[20] V. Komornik, Exact controllability and stabilization, RAM: Research in Applied Mathematics, Masson, Paris, 1994.

[21] J. Prüss, On the spectrum of $C_{0}$-semigroups, Trans. Amer. Math. Soc. 284 (1984), no. 2, $847-857$.

[22] F. L. Huang, Characteristic conditions for exponential stability of linear dynamical systems in Hilbert spaces, Ann. Differential Equations 1 (1985), no. 1, 43-56.

(C) 2017 by the authors; licensee Preprints, Basel, Switzerland. This article is an open access article distributed under the terms and conditions of the Creative Commons by Attribution (CC-BY) license (http://creativecommons.org/licenses/by/4.0/). 